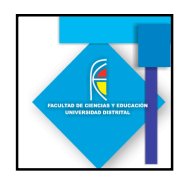

\title{
CONSTRUCCIÓN DE LAS ECUACIONES EN LA CIENCIA FÍSICA
}

\section{CONSTRUCTION OF THE EQUATIONS IN PHYSICAL SCIENCE}

\author{
Carlos Arturo Holguín Tabares ${ }^{1}$
}

\section{Resumen}

¿Cómo se obtienen las "formulas" de la física? No hay respuesta simple o única, porque construir las ecuaciones algebraicas con las reglas de composición que asocian magnitudes experimentales cuando cambia o varía una en función de la otra durante un experimento, requiere de un proceso lógico y consciente; a partir de definir el concepto de variable experimental y demás magnitudes presentes en fenómenos físicos, lo cual posibilita estudiar diferentes procedimientos debidamente argumentados. Algunos de estos métodos que relacionan las variables son: el análisis gráfico con diferentes papeles, las regresiones, el uso de leyes de conservación, análisis dimensional y otros, para construir ecuaciones algebraicas.

Palabras clave: Variable física, ecuación algebraica, análisis grafico, regresión.

\begin{abstract}
How are the physics equations gotten? there is not an only simple answer, because to construct the algebraic equations with the composition rules in experimental quantities associate; when one of them change in function of the other during an experiment, requires a conscious and logical process, since this to define the concept of experimental variables and the other more magnitudes present in physics phenomenons, it gives us possibilities to study different duly argue process. some of these methods that connect the variables are: graph analysis with different papers, use of the conservation rules, regression analysis and other to construct algebraic equations.
\end{abstract}

Keywords: Physical variable, algebraic equation, graphical analysis, regression.

"El resultado del descubrimiento de que vale la pena volver a comprobar por nueva experiencia directa y no confiar necesariamente en la experiencia del pasado" definición de ciencia del notable físico Richard Feynman [1].

\footnotetext{
${ }^{\text {I}}$ Profesor titular Departamento de Física, Universidad Tecnológica de Pereira. holguin@utp.edu.co
} 
$5^{\circ}$ CONGRESO NACIONAL DE ENSEÑANZA DE LA FÍSICA.

Universidad Pedagógica Nacional (U.P.N)

Universidad Distrital Francisco José de Caldas (U. D. F. J. C)

Bogotá, Colombia. 16 al 20 de mayo 2011

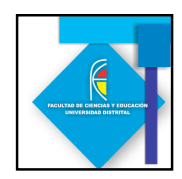

\section{Análisis gráfico}

En la realización de un experimento, se explora cuidadosamente que cantidades varían para identificar, aquellas magnitudes Físicas que cambian, o toman la forma de variables fundamentales de la Física, como se transforman; como se manifiestan, porque se dan, igualmente quien las genera y definir que magnitudes podrían ser variables pero que durante alguna parte o durante todo el experimento toma un solo valor, cantidad nombrada como parámetro. Establecer variables frecuentes de encontrar en los experimentos, cantidades provenientes de mediciones de: tiempo longitud, área, superficie, masa, temperatura, intensidad de corriente, intensidad luminosa, cantidad de sustancia, velocidad, aceleración, fuerza, energía y muchas otras más.

\begin{tabular}{|l|c|c|c|c|c|c|c|}
\hline $\mathrm{V} 1$ (Unidad de medida) & $\mathrm{V}_{1}$ & $\mathrm{~V}_{2}$ & $\mathrm{~V}_{3}$ & $\mathrm{~V} 1_{4}$ & $\mathrm{~V} 1_{5}$ & $\cdots$ & $\mathrm{V}_{\mathrm{n}}$ \\
\hline $\mathrm{V} 2$ (Unidad de medida) & $\mathrm{V} 2_{1}$ & $\mathrm{~V} 2_{2}$ & $\mathrm{~V} 2_{3}$ & $\mathrm{~V} 2_{4}$ & $\mathrm{~V} 2_{5}$ & $\cdots$ & $\mathrm{V} 2_{\mathrm{n}}$ \\
\hline
\end{tabular}

Tabla 1.1 Cuadro genérico con los posibles valores de las variables V1 y V2.

El experimentador luego de haber montado el equipo, inicia la práctica de laboratorio y toma medidas cuidadosamente de las variables V1 y V2, que consignará ordenadamente en tablas diseñadas y elaboradas previamente. Los valores experimentales

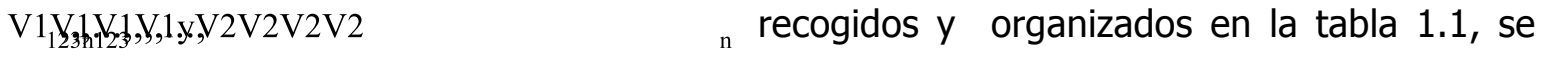
examinan para identificar, primero cuales son los valores extremos (limites inferior y superior) de todas las medidas registradas en cada variable y posteriormente al estudiar todos los valores por separado se evalúa, "sí cada valor posterior es mayor que el anterior y ese comportamiento se mantiene se tendrá una variable creciente y se representa: SivdldrdshyldpalowescRECIENTE ; ahora sí cada valor posterior examinado es menor que el anterior y además continua así, se tendrá una variable decreciente y se

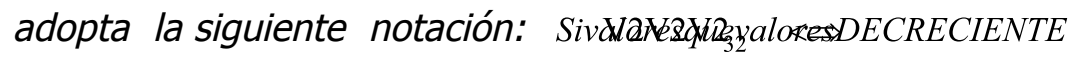

Posteriormente se evalúa el ¿Cómo cambian entre sí las dos variables registradas y tabuladas?, es decir cuando crece una de las variables ¿que le sucede a la otra? por ejemplo, sí V1 incrementa, entonces que le sucede a V2, ¿también se incrementará o se decrementará lo cual se nota: sí V1 $\$ z$ y viceversa sí V1 decrece entonces que le pasará a la otra V2? y se puede denotar V1 $\$ 23$ Si a través del examen preliminar se observa que cuando V1 crece, igualmente lo hace V2, en la misma proporción, se representará así: $S i \mathrm{~V} 1 \mathrm{y} \hat{\mathrm{N}} \mathbf{2}$, se presume razonablemente entonces una proporcionalidad directa, lo que constituye un análisis inicial simple que adquiere la categoría de hipótesis la cual debe ser verificada posteriormente. Sobre papel cuadriculado se construye un sistema de coordenadas rectangulares de dos dimensiones con ejes perpendiculares entre sí: uno vertical o eje de las ordenadas y otro horizontal o eje de las abscisas. El punto donde se intersecan los ejes perpendiculares se denomina origen de coordenadas y generalmente se le identifica con la letra $O$, el sistema coordenado produce cuatro cuadrantes. Sobre cada uno de los ejes se marcan divisiones, algunas con raya y número convencionalmente tomadas positivas desde el origen de coordenadas tanto hacia la derecha como en dirección perpendicular hacia arriba de la pagina y negativas desde el origen direcciones contrarias; luego se ubica sobre el eje horizontal la variable V2 y en el vertical los valores de V1 con sus correspondientes 


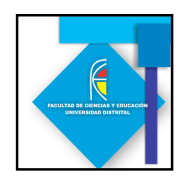

unidades. La figura simple o compleja construida con datos experimentales, ocurre cuando han sido localizados los puntos en el plano, los cuales dan lugar a una gráfica que puede ser una línea recta o curva, constituida por los pares ordenados $\left(\mathrm{V} 2, \mathrm{~V} 1_{2}\right) ;(\mathrm{V} 2, \mathrm{~V} 1) ; \ldots ;(\mathrm{V} 2, \ldots, \mathrm{V} 1) \quad$,que satisfacen la función $\mathrm{V} 1 \neq \mathcal{H}(\quad)$ Donde se interpreta que la línea construida es el lugar geométrico de los puntos que cumplen con la relación establecida entre las variables V1 y V2.

\section{Funciones lineales, la línea recta}

De los pares ordenados como datos experimentales; resulta razonable suponer que sí al graficarlos reproducen una línea recta, tales valores asociados con las variables V1 y V2 permiten asegurar que alcrecer $\mathrm{V} 1 \widehat{ } \overrightarrow{2} \Rightarrow \uparrow \quad$ en la misma proporción, permite plantear la hipótesis de que "existe una proporcionalidad directa entre dichas variables cuya representación: V1æ2 " corresponde al único caso donde se lee que " la variable V1 es

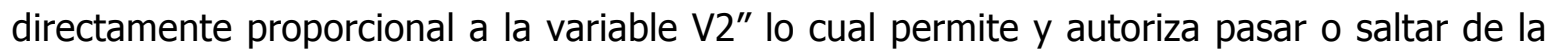
proporcionalidad directa a la construcción de la ecuación V1€teV2 Que es la ecuación de la línea recta que pasa por el origen y se prueba la hipótesis al evaluar nuevos puntos no experimentales que satisfagan la ecuación anterior. El tipo de función que relaciona a V1 con V2 se conoce como función lineal y en general toma la forma V1€车V2b , donde de dicha ecuación se desprenden las siguientes preguntas: 1 ¿Cuánto vale la constante? 2 ¿Quién es y que representa dicha constante? De la ecuación general surge el interrogante 3 ¿quien es $\pm \mathrm{b}$ y como se interpreta experimentalmente? La pregunta 1 se resuelve calculando la pendiente de la recta, o tomando la tangente del ángulo formado por la recta graficada con la horizontal como se ilustra en la figura 1.1

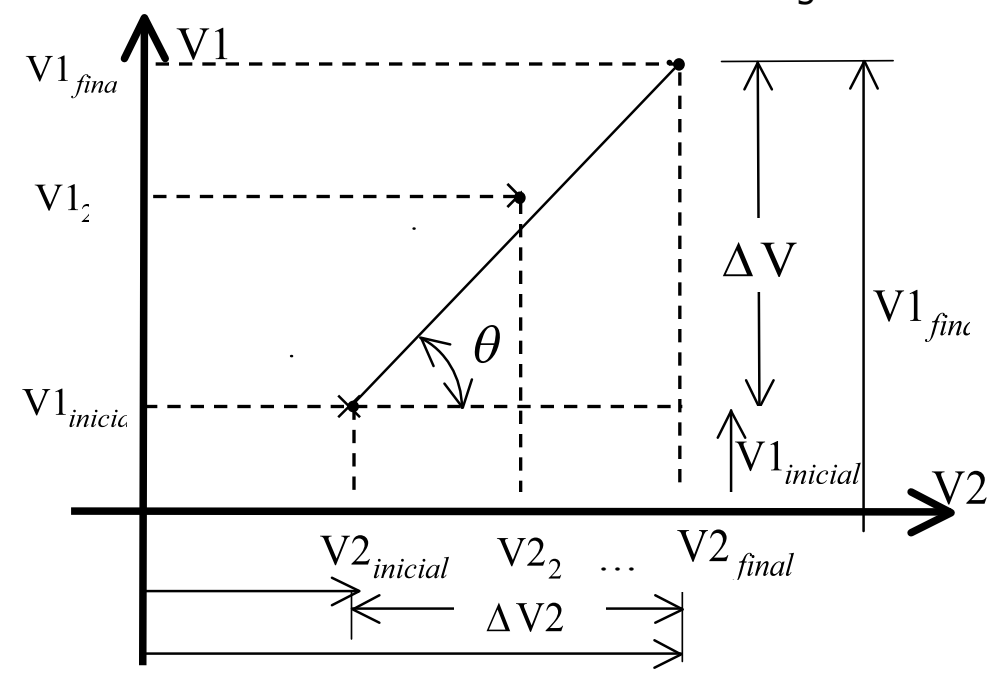

Figura 1.1 Grafica que ilustra el método para determinar la pendiente de la recta experimental.

$$
\tan \theta=\frac{\Delta \mathrm{V} 1}{\Delta \mathrm{V} 2 \mathrm{~V} 2 \mathrm{~V} 2_{\text {(finali)nicial }}(\quad)} \quad \text { Cte }
$$


$5^{\circ}$ CONGRESO NACIONAL DE ENSEÑANZA DE LA FÍSICA.

Universidad Pedagógica Nacional (U. P. N)

Universidad Distrital Francisco José de Caldas (U. D. F. J. C)

Bogotá, Colombia. 16 al 20 de mayo 2011

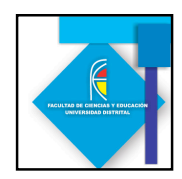

Las respuestas 2, 3 y 4 (sí éste último existe) están íntimamente ligadas al experimento particular realizado, siendo normalmente condiciones iniciales del problema estudiado.

\section{Funciones no lineales}

Algunas curvas familiares, que no son líneas rectas se pueden convertir en lineales, tratadas por medio de la técnica de cambio de variable; un número considerable de fenómenos y leyes físicas, se expresan a través de funciones del tipo V1€Vał n , en lo sucesivo se reemplaza la $C t e$ por la letra " $a$ " y la expresión algebraica adoptada por

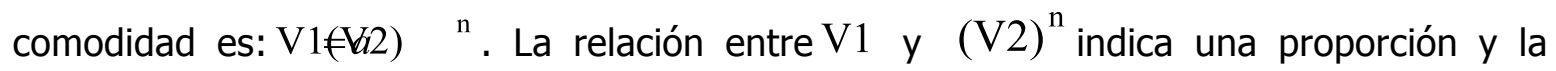
magnitud de " $a$ " se reconoce como constate de proporcionalidad; para casos donde $\mathbf{n}$ es negativo se denominan variables inversamente proporcionales, la notación tiene la forma $\mathrm{V} 1 \neq \mathrm{V} 2) \quad{ }^{-1}$ o su forma equivalente $\mathrm{V} 1=a 1 / \mathrm{V} 2$; con $\mathrm{n} z$, una parábola se dice que son proporcionales con el cuadrado y la notación es V1fVa2) 2 ; para $n \theta, 5$ se denominan proporcionales a la raíz cuadrada y se representa $\mathrm{V} 1(\mathrm{~V} a) \quad 0,5$. Un caso importante en la física tiene la forma V1f 62$)^{-2}$ se dice "la variable V1 es proporcional con el inverso cuadrado de la variable V2 "

\section{Regresiones}

Método analítico basado en trabajo y calculo estadístico, también llamado ajuste de curvas por mínimos cuadrados, siendo el caso más conocido y simple la ecuación de la línea recta que mejor representa un conjunto de datos experimentales, también contempla la construcción de las ecuaciones algebraicas de regresiones no lineales.

\section{Regresión lineal}

Al graficar variables sobre un plano, los lugares geométricos reproducen rectas y muchas rectas podrían representar los puntos, ¿Cuál es la recta correcta? Lo esperable es emplear un procedimiento matemático, que evitaría la inseguridad de un juicio subjetivo. El procedimiento se basa en el principio estadístico de los mínimos cuadrados y se aplica en forma restringida para encontrar una línea recta que se ajuste a los valores medidos. Se supone un conjunto de " $n$ " valores de una variable $V 1$, medidos como función de la variable V2 y toda la incertidumbre concierne a la dimensión V1, esto es, los valores de V2 se conocen con una precisión mucho mayor que los de V1. Si no es así, el tratamiento no será valido y el método requerido es otro. Nuevamente en el plano V2 - V1, "Cual es la mejor recta"? El principio de mínimos cuadrados permite la escogencia sobre la base de las desviaciones de los puntos $\mathrm{CH}_{i}$ tomados en la vertical a partir de la posible recta escogida; entonces la recta mas representativa es aquella que minimiza la suma de los cuadrados de las desviaciones verticales $\Sigma\left(\mathrm{CH}_{i}\right)^{2}$ y se toma éste criterio para optimizar la 


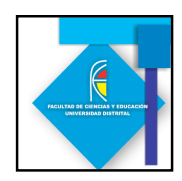

trayectoria de línea propuesta entre tales puntos. Se calcula la pendiente $a$ y la vertical desde el origen hasta el corte con la recta $b$, de la recta. Considérese como la ecuación de la recta: $\mathrm{V} 1=\mathrm{d} B \mathrm{~B} \pm$, entonces la magnitud de la desviación $\mathrm{Ch}_{i}$ es el intervalo entre un cierto valor medido $\mathrm{V} 1_{i}$ para el valor de $\mathrm{V} 2 ;$ y se puede calcular a partir del valor correspondiente de $\mathrm{V} 2$; dado $a \mathrm{~W} 2_{i}{ }+$, si se llama $\delta \mathrm{V} 1_{i}$, cada diferencia se tendrá $\delta \mathrm{V} 1 \mathrm{~W} \in \mathrm{W} \mathrm{W} 2(a b \quad)$. El criterio de mínimos cuadrados permite calcular los valores

deseados de $a$ y $b$, a partir de la condición, $\Sigma\left[\forall y_{i l} V_{2} \text { Abbínimo }\right]^{2} \quad$ entonces, $\frac{\partial \mathbb{M i n M i n}}{\partial \bowtie b}==$ yo

Un breve ejercicio algebraico permite obtener los valores buscados de la pendiente y de ordenada al origen de la mejor línea, aplicando las siguientes ecuaciones:

\begin{tabular}{|c|c|c|c|c|}
\hline \multicolumn{3}{|c|}{ 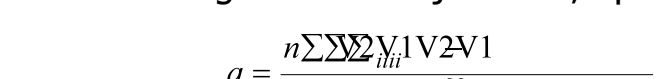 } & \multicolumn{2}{|c|}{ 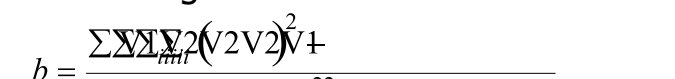 } \\
\hline & $\left.n \sum x(\mathrm{~V} 2 \mathrm{y})\right)^{22}-($ & ) & $n \sum X(\mathrm{~V} 2 \mathrm{y}) \mathrm{e}^{22}-($ & 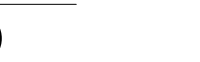 \\
\hline Medida No. & $\mathrm{V} 2(1 \otimes \mathrm{A}) \quad-3$ & $\mathrm{~V} 1 \Downarrow V()$ & $(I(\mathrm{~A}))^{2}$ & $I Y \mathrm{~A})(\mathrm{V})$ \\
\hline . & . & & & \\
\hline $\mathrm{n}=$ & $\Sigma \neq\left(10 \AA^{3}\right)$ & $\sum \neq(\mathrm{V})$ & $\Sigma \varsubsetneqq I(\mathrm{~A})]^{2}$ & $\Sigma \neq(\mathrm{A})(\mathrm{V})$ \\
\hline
\end{tabular}

Tabla 1.2 Valores del experimento real para verificar la ley de ohm.

Se controla la apreciación subjetiva de quien evalúa la información experimental, con un procedimiento matemático, con valores cercanos a de verdad y de gran acogida por investigadores casi en forma universal. La tabla 1.2 facilita la recolección de información experimental, incluye las operaciones a realizar, indicadas en la parte superior de las columnas que proveen la información para calcular los parámetros $a y b$.

\section{Regresión no lineal}

Variables con exponente diferente a la unidad se tratan con los mismos principios y procedimientos de la regresión lineal, la diferencia estriba en que el logaritmo natural "In" actúa sobre cada variable para generar los nuevos valores de la tabla 1.3, donde se realizan los cálculos indicados en la primera fila, cantidades que permiten obtener las sumas de la ultima fila para reemplazar en las ecuaciones para el calculo de los parámetros, no obstante ser idénticas en estructura a las empleadas en la regresión lineal, aquí se trabaja el logaritmo natural de la variable y como no son valores absolutos se recomienda trabajar con cuatro decimales por ser logaritmos.

$[\mathrm{V} 1 \mathrm{ll}] \# 1 \quad[\mathrm{~V} 2 \mathrm{l}] \sharp 2$ 
$5^{\circ}$ CONGRESO NACIONAL DE ENSEÑANZA DE LA FÍSICA.

Universidad Pedagógica Nacional (U. P. N)

Universidad Distrital Francisco José de Caldas (U. D. F. J. C)

Bogotá, Colombia. 16 al 20 de mayo 2011
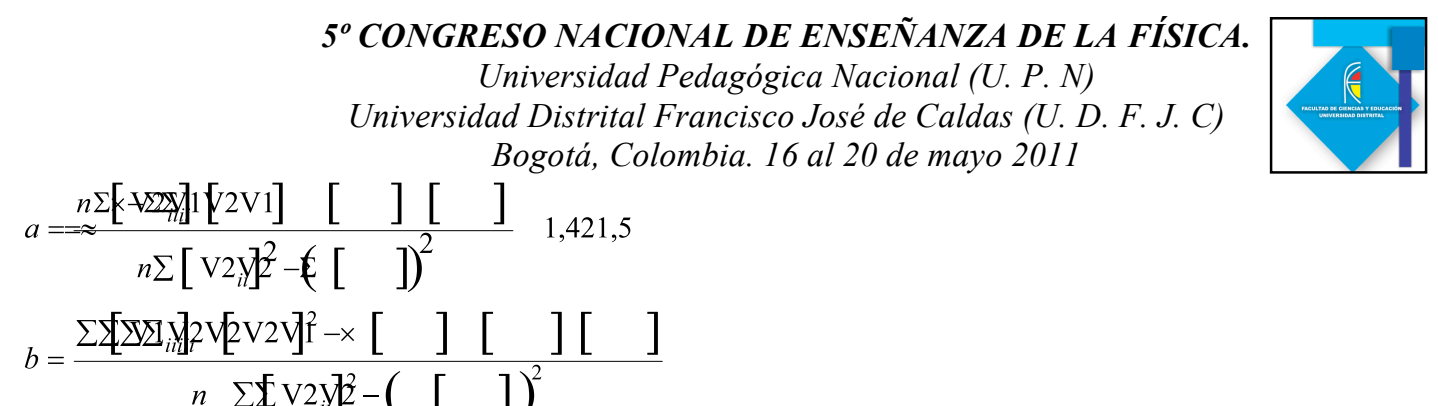

\begin{tabular}{|c|c|c|c|c|c|c|}
\hline Medida $\mathrm{N}_{0}$ & $\mathrm{~V} 1(\pitchfork)$ & $\mathrm{V} 2(\mathrm{~m} X)$ & {$[V I]=\ln$} & {$[I I]=\ln$} & {$\left[\|^{2}\right.$} & {$[M] \times[]$} \\
\hline$\cdot$ & $\cdot$ & $\cdot$ & $\cdot$ & $\cdot$ & $\cdot$ & $\cdot$ \\
$\cdot$ & $\cdot$ & $\cdot$ & $\cdot$ & $\cdot$ & $\cdot$ & $\cdot$ \\
\hline$n=$ & & & $\Sigma[V]=$ & $\Sigma[I]=$ & $\Sigma[]^{2}=$ & $\Sigma[M] \times[]=$ \\
\hline
\end{tabular}

Tabla 1.3 Cuadro genérico de datos para construir una ecuación no lineal.

El resultado final entonces será la nueva ecuación, que surge del posterior cálculo de los parámetros $a$ y $b$ luego de calcular la constante $k$ para darle la forma definitiva a la ecuación buscada $k e={ }^{b} \quad \operatorname{V1} \forall 2 k()^{a}$

\section{Uso del papel log-log}

En el papel logarítmico se obtienen directamente los valores de " $n$ " y " $a$ ", sin necesidad de hacer múltiples ensayos para ello, al partir de la función: $\operatorname{V1} ¥ 2()^{n}$ donde cualquier operador que actué lo hará afectando todos los términos de la expresión. Al tomar logaritmos a ambos lados de la ecuación, resulta: $\log \left(1 \operatorname{lo} \$ \sharp 2\left(a()^{n}\right)\right.$ y $\log (1 \log$ logVan ( ). Graficar sobre papel logarítmico variables de una tabulación, siempre conduce a una recta, que la mayoría de las veces no pasará por el origen, salvo cuando la constante de proporcionalidad valga uno, y cuya pendiente es el valor de " $n$ ". Se colige de la última ecuación que la ordenada al origen es " $a$ " y al emplear papel logarítmico y trazar sobre él, las parejas ordenadas, se advierte, que el papel logarítmico da la escala en que se dividen los ejes V2 eje horizontal y V1 vertical, por lo cual no es valido alterarla, además, su uso se requiere tantos ciclos como cifras por décadas existan entre los extremos de los valores experimentales de cada variable; verbigracia si los datos de una variable comprenden valores desde la décimas hasta las centenas necesitara: un ciclo para décimas otro para unidades otro para decenas, y otro centenas. En general se exploran los valores extremos de las variables $\mathrm{V} i$; por encima $\mathrm{V} i_{\text {máx }} \mathrm{y}$ por debajo $\mathrm{V} i_{\text {min }}$ e informa cuantos ciclos asociados a décadas necesita el conjunto de datos para aplicar en el eje respectivo.

\section{Papel milimetrado para graficar el logaritmo de las variables}

Esta técnica permite graficar los logaritmos naturales de las variables experimentales V1 y V2 sobre papel milimetrado y a partir de la recta construida generar la ecuación 


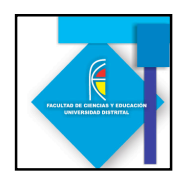

respectiva. Graficar los logaritmos conducen a la recta, que casi nunca pasa por el origen, salvo cuando la constante de proporcionalidad " $a$ " valga uno, la cual se calcula siguiendo el método de la figura 1.1, al trabajar con papel milimetrado se debe primero encontrar el logaritmo natural de los datos y registrarlos en un cuadro; la intención es que se pueda relacionar las variables experimentales para concluir en una expresión algebraica lógica con la forma de la función: $\mathrm{V} 1 \supsetneqq 2 k()^{a}$, se colige de la ecuación que la ordenada al origen $k$ se corresponde en la grafica con $k=\operatorname{Ln}($ magnituddelaordenadaalorigen) .

\section{Referencias Bibliográficas}

Baird, D.C. (1988). Evaluación de experimentos. Una introducción a la teoría de mediciones y al diseño de experimentos, Mexico: Prentice-Hall Hispanoamericana S.A.

Fishbane, P. Gasiorowicz, S. Thornton, S. (1994). Fisica para ciencias e ingenieria. Mexico: Prentice-Hall Hispanoamericana, S.A.

Halliday, D. Resnick, R., Krane, K. (2002). Fisica. Mexico: Grupo Patria Cultural S.A.

Holman, J.P. (1986). Metodos experimentales para ingenieros. Mexico:McGraw-Hill.

Levens, A. (1972). Análisis Gráfico : para arquitectura e Ingeniería. Mexico: Limusa-Wiley.

Sears, F. Zemansky, M. Young, H. Freedman, R. (2009). Fisica universitaria. Mexico: Fondo Educativo Interamericano. S.A. 